\title{
Repair or replacement of restorations: do we accept built in obsolescence or do we improve the evidence?
}

\author{
IN BRIEF \\ - Demonstrates the evidence base for the \\ replacement and repair of amalgam and \\ resin composite restorations enabling \\ practitioners to make an evidence-based \\ decision. \\ - Discusses the implications of the \\ evidence on clinical practice. \\ - Discusses the implications of current \\ evidence on future research.
}

\author{
M. O. Sharif, ${ }^{1}$ Z. Fedorowicz, ${ }^{2}$ M. Tickle ${ }^{3}$ and P. A. Brunton ${ }^{4}$
}

VERIFIABLE CPD PAPER

\begin{abstract}
Introduction Controversy surrounds decisions on whether to repair or replace defective dental restorations. The concept of built-in obsolescence, ie periodic replacement of dental restorations, is largely accepted as the modus operandi if not the default mode in restorative care. In this article we examine the current best available evidence underpinning the effectiveness of replacement versus repair of direct amalgam and resin composite restorations. Method This article builds on two recent Cochrane systematic reviews which have reported on the evidence base supporting the effectiveness of replacement versus repair of amalgam and direct resin composites and translates the results of their research conclusions into recommendations for the dental clinician. Results As no relevant randomised controlled trials (RCTs) were identified in either of these systematic reviews, the evidence regarding the effectiveness of repair versus replacement of amalgam and composite restorations is weak and incomplete. The evidence as it currently stands seems to favour repair over replacement, but this is insufficient to make firm recommendations. Conclusion In view of the absence of high level evidence there is a need for further well designed RCTs. To add value to the evidence base these trials should be conducted in a general practice setting which will strengthen the generalisability and applicability of the research conclusions and enable dentists and patients to make informed decisions.
\end{abstract}

\section{INTRODUCTION}

In the UK amalgam continues to be the most popular option ${ }^{1}$ to restore posterior teeth, although its use has declined recently, and there has been a corresponding steady increase in the use of direct composite resin restorations. ${ }^{2,3}$ Considerable resources in terms of time and money are spent restoring teeth $\mathrm{h}^{4-6}$ and as restorations have a limited lifespan periodic costly interventions are required eg patch and repair or even complete replacement of restorations. With each intervention less and less tooth structure remains and eventually the tooth may become unrestorable or even suffer catastrophic fracture and may need to be extracted. This serial replacement

"NIHR In-Practice Research Fellow, ${ }^{3}$ Professor of Dental Public Health and Primary Care, Oral Health Unit, School of Dentistry, University of Manchester, Manchester, M15 6LP; ${ }^{2}$ Director - The Bahrain Branch of the UK Cochrane Centre, The Cochrane Collaboration; ${ }^{4}$ Professor of Restorative Dentistry, Leeds Dental Institute, University of Leeds, Leeds

*Correspondence to: Mr Mohammad Sharif Email: mohammad.owaise.sharif@googlemail.com

\section{Refereed Paper}

Accepted 30 April 2010

DOI: 10.1038/sj.bdj.2010.722

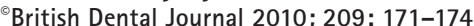

of restorations by ever larger and more complex restorations has been called the restorative cycle, spiral or staircase. ${ }^{7}$

Although recurrent caries is still considered to be the most common reason for the failure of amalgam restorations, ${ }^{8-12}$ fracture of the filling or the tooth itself are also considered to be major causes. ${ }^{11,13-18}$ Composite resin restorations fail for many of the same reasons although poor aesthetics as a consequence of material degradation and discolouration has been highlighted as one of the principal reasons which are important to patients. ${ }^{19}$ Other factors that result in the replacement of resin composite restorations include the loss of marginal integrity due to breakdown often causing pain and discomfort. ${ }^{20}$

The traditional and possibly most widely accepted treatment option for a defective restoration has favoured total replacement rather than their repair. ${ }^{21}$ However, a critical and at times difficult balance needs to be struck between the perceived benefits and the potential harm of each of these options. The unwarranted replacement of a restoration can result in not only a substantial loss of tooth tissue $e^{22-24}$ but may also lead to additional and unnecessary insult to the pulp. The often less traumatic approach of repair may be perceived as being more acceptable by patients, whereas clinicians may see this as a financially less attractive option or indeed even consider it to be substandard care.

Although the most common reason for the replacement of any restoration is recurrent caries, the term 'recurrent caries' remains ill-defined. ${ }^{25}$ It can encompass extensive carious lesions which have completely undermined a restoration in which the only solution is the removal of caries and complete replacement of the restoration; minimal carious lesions adjacent to restoration margins and also non-carious defects adjacent to restorations. ${ }^{26}$ Often restorations with a marginal gap, a fracture or with an associated tooth fracture but without any evidence of caries are included under this catch-all term. These scenarios often provide dilemmas for clinicians in deciding whether some of these lesions are treated more appropriately by means of repair rather than total replacement. The repair of restorations is in general considered a more conservative 
treatment option, which in addition to saving time may increase the long term survival of the tooth, and in some situations may be performed without the use of local anaesthesia and therefore prove less distressing for patients. ${ }^{27}$

Assessment of the integrity of a restoration can be quite complex, tending to be subjective and therefore defective restorations which might conceivably be best treated by repair may often simply be replaced. ${ }^{28}$ This subjective nature of clinical decision making can lead to large variation in the care provided by different clinicians for the same condition, resulting in concerns about the quality of care provided. A wide range of validated and internationally recognised scales which are capable of evaluating the clinical acceptability of resin composite restorations are available, ${ }^{29-31}$ but many of these are not easy to use in a general dental practice setting. ${ }^{29-31}$ Other factors that may influence treatment decisions are the system of remuneration, ${ }^{32}$ for example a fee per item system may encourage total replacement of restorations whereas capitation-based systems may encourage repair because finance is secured regardless of treatment modality and repairs generally take less time and possibly consume fewer materials resulting in lower direct costs. It is well recognised that treatment decisions by clinicians are influenced by a complex and varied range of factors which are not solely based on knowledge and technical skills but include cognitive behaviour, perceptions and individual attitudes. ${ }^{33}$ It has been suggested that generational difference may affect treatment decisions as traditional teaching has favoured the replacement of defective restorations as the ideal treatment ${ }^{21}$ and so older dentists may favour replacement over repair.

This paper presents a summary of the currently available evidence for the effectiveness of replacement versus repair of direct amalgam and resin composite restorations, discusses its strength and direction and illustrates the implications of that evidence on clinical practice.

\section{REPAIR OR REPLACEMENT OF DEFECTIVE RESTORATIONS - THE EVIDENCE}

Two recent Cochrane reviews ${ }^{34,35}$ sought to evaluate the evidence for the effectiveness of replacement versus repair of defective amalgam and resin composite dental restorations on a like for like basis in permanent molar and premolar teeth. The summary of that evidence was assembled through the use of explicit methods which included a systematic search for eligible studies and the critical appraisal and synthesis of relevant data extracted from those primary studies. Protocols which outlined a clear strategy of how the reviews were to be conducted, were developed and published for each of these reviews. ${ }^{36,37}$

These protocols included a priori statements which posed a clear research question and set out criteria which directed a comprehensive search for all relevant articles, and dictated the process of inclusion or exclusion of studies, which in the case of these Cochrane reviews only considered RCTs. The review process had clearly designated steps to: identify studies and the methods which were to be employed to assess their quality; guide the way in which data were to be extracted; and define the statistical techniques that would be used in the synthesis of the data. Transparency and reproducibility were ensured through the documenting of all decisions taken throughout this process and the reviews conclude with a summary of the results on which conclusions about the interventions were based.

A comprehensive electronic search of the literature which included the major healthcare databases (MEDLINE via OVID, CENTRAL, The Cochrane Library) was conducted. Search terms which were carefully selected to maximise the opportunity of identifying relevant RCTs were combined with phases 1 and 2 of the Cochrane Sensitive Search Strategy for RCTs (as published in the Cochrane handbook for systematic reviews of interventions). ${ }^{38}$ There were no language restrictions for inclusion in the reviews and studies were translated as required.

The total number of potentially eligible trials retrieved in both searches was 117 (resin 73, amalgam 44). After preliminary assessment of the titles and abstracts all but four studies were excluded from further evaluation; the principal reason for exclusion was failure to randomise patients into treatment groups. Full text copies of the remaining studies ${ }^{28,39-41}$ were obtained and these then underwent further evaluation against the pre-specified inclusion criteria for this review. The bibliographic references in these papers were also examined for other potentially eligible studies, but no further studies were identified. Three of the remaining studies ${ }^{39-41}$ were common to both resin composites and amalgam, whereas only one study ${ }^{28}$ investigated composite resins.

None of the included studies were able to provide reliable evidence for the effectiveness of replacement versus repair of resin composite and amalgam restorations. One study by Gordon ${ }^{28}$ used inappropriate randomisation of its participants and therefore resulted in its exclusion from the review. Moncada ${ }^{40}$ and Moncada ${ }^{39}$ reported on the same set of patients but at different follow up periods. In both of these studies the patients were reported to have been randomly allocated to treatment groups but the method of randomisation was not reported. Inadequate and incomplete reporting of the results in these studies meant that the data were unusable and attempts to contact the authors for clarification proved unsuccessful. In Moncada ${ }^{41}$ patients were not randomly allocated to treatment groups but were assigned into groups according to defect type and therefore this study was also excluded from the review.

\section{Summary of evidence base}

Although none of the studies retrieved for both of these reviews fully matched the inclusion criteria and cannot be described as methodologically sound trials with a low risk of bias, the results reported in these studies do suggest that repair of restorations may prove to be as effective a treatment option as total replacement. The survival rate of repairs at three years was as good as total replacement, ${ }^{41}$ with no failures in the repair group. This information needs to be considered alongside the fact that repairs can often be completed without the use of local anaesthesia and are therefore less distressing for a patient when compared with total replacement with local anaesthesia.

\section{DISCUSSION}

Dental healthcare professionals are increasingly aware that if they are to ensure the delivery of the best possible level of care that their clinical decision making should 
be based on the current best evidence of effectiveness of the interventions they provide. Evidence based medicine/dentistry can be defined as "the integration of the best research evidence with clinical expertise and patient values'. ${ }^{43}$ This can also be explained in the following terms: the integration of clinical expertise with the best available evidence while fully taking into account the patient's wishes thereby providing effective and efficient diagnosis and prescription of the most appropriate treatment modality for individual patients. Although it is frequently referred to in the literature as a 'new paradigm' the concept of evidence-based healthcare is not new, the literature on this topic is extensive and its pervasive influence has been felt across healthcare delivery over the last two decades. Its importance was most recently highlighted in the Steele review $^{42}$ of the 2006 NHS dental contract which recommended care should be delivered via evidence-based care pathways following a detailed, structured assessment of each patient.

After many years of acceptable service a restoration and/or its interface with the tooth may become deficient. This can be due to a number of reasons, for example wear, fatigue, caries, and fracture of the tooth or the restoration. The risk of these processes varies from patient to patient with variables such as extent of bruxism and level of oral hygiene playing a key role. The current available evidence from longitudinal observational studies suggests that repairing restorations have short term survival rates similar to total replacement. ${ }^{41}$ This information allied to a perception that repairs are less invasive for patients and consume less resources support a provisional view that GDPs should favour repair over replacement.

Clinical procedures with respect to the repair and replacement of restorations have largely evolved in a piecemeal and anecdotal way and there is little understanding of how widely repair of restorations has been adopted. It is clear that practitioners do repair restorations but what factors they consider when deciding to repair or replace a restoration are unclear. It is generally accepted that restorations are repaired with the same material and that amalgam repairs are mechanically keyed into the remaining restoration or tooth structure while resin composite restorations are repaired after the surface of the remaining restoration has been prepared to remove contamination. Little is known about the efficacy of bonding new resin composite to an existing restoration but anecdotally, repairs to existing resin composite restorations seem to perform well. Resin composite restorations are often resurfaced and refinished and this is distinct from a repair, but certainly the concept of a serviceable restoration is generally accepted for resin composite as distinct from amalgam restorations. Preventive dentistry has mainly been associated with the prevention of the two major dental diseases, however, the repair of a defective restoration as an alternative to its total replacement (where possible) is seen as an increasingly important preventive procedure because it minimises loss of tooth tissue.

For an appropriate treatment decision to be made when faced with a defective restoration the dentist must combine clinical expertise (including correct diagnosis and consideration of individual patient factors such as oral hygiene, motivation, aesthetic and financial concerns) with the patient's choice. A patient will only be able to make a truly informed decision (choice) if the dentist has explained all appropriate treatment options and the associated risks. The patient should be made aware of restorative staircase and the potential harm caused by replacement where repair is possible as well as the uncertainty regarding the longevity of repairs and the associated financial implications. The current state of the evidence base (and its shortcomings) underpinning such treatment options should be fully discussed.

Restoration of teeth is one of the mostly commonly provided (and therefore costly) treatments provided by dentists. With improving dental health ${ }^{4,44}$ the majority of restorative care provided in the UK over the next 30 years or so will be restoration of defective restorations. There is a lack of firm evidence on whether repair or replacement is better for patients but this should not be interpreted as meaning that repair of defective restorations does not work. Indeed the evidence as it stands tends to favour repair over replacement. However, there is an urgent need for methodologically sound, large randomised controlled trials to be conducted on this topic. Such trials raise significant challenges for dental researchers; they should be conducted in general dental practice as the majority of care is provided by GDPs and we need to know what works best in their hands. The studies should measure mechanical failure and also explore costs and evaluate important patient reported outcome measures such as peri- and post-operative pain, anxiety and distress. All of the studies retrieved had a medium-term (less than five years) follow up; it would be beneficial if future trials had a longer term follow up (five years and above), but this would increase the risk of loss to follow up in the trial. The longitudinal studies suggest little difference between repair and replacement which means that large numbers of participants would be required to detect a significant difference. All of these factors mean that a trial would be very expensive, but the costs of a trial should be evaluated with reference to the hundreds of millions of pounds spent each year on restorations. Perhaps in the short term a consensus view from experts should be produced to help inform the decision making of GDPs in line with the requirements of the Steele review $^{42}$ to produce clinical pathways.

\section{CONCLUSION}

The evidence regarding the effectiveness of repair versus replacement of amalgam and composite restorations is weak and incomplete. Flawed data suggest repairs have similar short term survival to replacement and are less invasive. There is an urgent need for well designed randomised controlled trials to provide strong evidence to inform clinical practice. In addition further research needs to be undertaken that focuses on helping dentists accurately and reliably assess the integrity of restorations allied to national guidelines based on expert consensus to enable them to decide if and when a restoration needs to be repaired or replaced.

Mohammad Owaise Sharif is a National Institute for Health Research (NIHR) In-Practice Research Fellow.

1. Wilson N H F, Christensen G J, Cheung S W, Burke F J T, Brunton P A. Contemporary dental practice in the UK: aspects of direct restorations, endodontics and bleaching. Br Dent J 2004; 197: 753-756.

2. Lynch C D, McConnell R J, Wilson N H F. Teaching of posterior composite resin restorations in undergraduate dental schools in Ireland and the United Kingdom. Eur J Dent Educ 2006; 10: 38-43.

3. Opdam N J M, Loomans B A C, Roeters F J M, Bronkhorst $\mathrm{E}$ M. Five year clinical performance of 
posterior composite resin restorations placed by dental students. J Dent 2004; 23: 379-383.

4. Kelly M, Steele J G, Nuttall N et al. Adult Dental Health Survey. Oral Health in the United Kingdom 1998. 2000.

5. Qvist V, Thylstrup A, Mjör I A. Restorative treatment pattern and longevity of amalgam restorations in Denmark. Acta Odonto/ Scand 1986; 44: 343-350.

6. Qvist V, Thylstrup A, Mjör I A. Restorative treatment pattern and longevity of resin restorations in Denmark. Acta Odontol Scand 1986; 44: 351-359.

7. Brunton P A, Kay E J. Prevention. Part 6: Prevention in the older dentate patient Br Dent J 2003; 195: 237-241.

8. Burke F J, Cheung S W, Mjör I A, Wilson N H. Reasons for the placement and replacement of restorations in vocational training practices. Prim Dental Care 1999; 6: 17-20.

9. Mjör I A, Moorhead J E, Dahl J E. Reasons for replacement of restorations in permanent teeth in general dental practice. Int Dent J 2000; 50: 361-366.

10. Mjör I A, Shen C, Eliasson S T, Richter S. Placement and replacement of restorations in general dental practice in Iceland. Oper Dent 2002; 27: 117-123.

11. Mjör I A, Toffenetti F. Placement and replacement of amalgam restorations in Italy. Oper Dent 1992; 17: 70-73.

12. Setcos J C, Khosravi R, Wilson N H, Shen $C$ et al. Repair or replacement of amalgam restorations: decisions at a USA and a UK dental school. Oper Dent 2004; 29: 392-397.

13. Friedl K H, Hiller K A, Schmalz G. Placement and replacement of amalgam restorations in Germany. Oper Dent 1994; 19: 228-232.

14. Bernardo $M$, Luis $H$, Martin M D, Leroux B G et al. Survival and reasons for failure of amalgam versus composite posterior restorations placed in a randomized clinical trial. J Am Dent Assoc 2007; 138: 775-783.

15. Burke F J T. Influence of patient factors on age of restorations at failure and reasons for their and replacement. J Dent 2001; 29: 317-324

16. Mjör I A, Gordan V V. Failure, repair, refurbishing and longevity of restorations. Oper Dent 2002 27: 528-534.

17. Mjör I A, Moorhead J E. Selection of restorative materials, reasons for replacement, and longevity of restorations in Florida. J Am Coll Dent 1998;
65: 27-33.

18. Klausner L H, Green T G, Charbeneau G T. Placement and replacement of amalgam restorations: a challenge for the profession. Oper Dent 1987; 12: 105-112.

19. Manhart J, Chen H Y Hamm G, Hickel R. Review of the clinical survival of direct and indirect restorations in posterior teeth of the permanent dentition. Oper Dent 2004; 29: 481-508.

20. Mjör | A. The reasons for replacement and the age of failed restorations in general dental practice. Acta Odontol Scand 1997; 55: 58-63.

21. Wilson N, Setcos J C, Brunton P. Replacement or repair of dental restorations. In: Advances in operative dentistry: contemporary clinical practice. Quintessence Publishing Co Ltd, 1999.

22. Gordon V V. In vitro evaluation of margins of replaced resin based composite restoration J Esthet Dent 2000; 12: 217-223.

23. Gordon V V. Clinical evaluation of replacement of class $\mathrm{V}$ resin based composite materials. J Dent 2001; 29: 485-488.

24. Gordon V V, Mondragon E, Shen C. Evaluation of the cavity design, cavity depth, and shade matching in the replacement of resin based composite based restorations Quintessence Int 2002; 32: 273-278.

25. Mjör | A. Clinical diagnosis of recurrent caries. J Am Dent Assoc 2005; 10: 1426-1433.

26. Mjör I A, Gordan V V. Failure, repair, refurbishing and longevity of restorations. Oper Dent 2002; 27: 528-534.

27. Mjör I A. Repair versus replacement of failed restorations Int Dent J 1993; 43: 466-472.

28. Gordan V V, Shen C, Riley J. Two-year clinical evaluation of repair versus replacement of composite restorations. J Esthet Dent 2006; 18: 144-154.

29. Sarrett D C. Clinical challenges and the relevance of materials testing for posterior composite restorations. Dent Mater 2005; 21: 9-20.

30. Ryge G. Clinical criteria. Int Dent J 1980; 30: 347-358.

31. Lindberg A. Resin composites: sandwich restorations and curing techniques. Odontologi, 2005.

32. Burke F J, Wilson N H, Cheung S W, Mjor I A. Influence of the method of funding on the age of failed restorations in general dental practice in the UK. Br Dent J 2002; 192: 699-702.

33. Forsetlund L, Bjørndal A, Rashidian A et al.
Continuing education meetings and workshops: effects on professional practice and health care outcomes. Cochrane Database Syst Rev2009: CD003030.

34. Sharif M O, Merry A, Catleugh M et al. Replacement versus repair of defective restorations in adults: amalgam. Cochrane Database Syst Rev 2010; CD005970.

35. Sharif M O, Catleugh M, Merry A et al. Replacement versus repair of defective restorations in adults: resin composite. Cochrane Database Syst Rev 2010; CD005971.

36. Merry A, Catleugh M, Tickle M, Brunton P, Dunne $S \mathrm{M}$. Replacement versus repair of defective restorations in adults: amalgam. Cochrane Database Syst Rev 2006; CD005970. .

37. Catleugh M, Merry A, Tickle M, Dunne S M, Brunton P A. Replacement versus repair of defective restorations in adults: resin composite (Protocol). Cochrane Database Syst Rev 2006; 3: 1-6.

38. Higgins J P T, Green S (eds). Cochrane handbook for systematic reviews of interventions. Version 5.0.2 [updated September 2009]. The Cochrane Collaboration, 2008. Available from www.cochrane-handbook.org.

39. Moncada G C, Martin J, Fernandez E et al. Alternative treatments for resin-based composite and amalgam restorations with marginal defects: a 12-month clinical trial. Gen Dent 2006 54: 314-318.

40. Moncada G, Fernandez E, Martin J et al. Increasing the longevity of restorations by minimal intervention: a two-year clinical trial. Oper Dent 2008; 33: 258-264.

41. Moncada G, Martin J, Fernandez E et al. Sealing, refurbishment and repair of Class I, Class II defective restorations: a three-year clinical trial. J Am Dent Assoc 2009; 140: 425-432.

42. NHS dental services in England. An independent review led by Professor Jimmy Steele. London: Department of Health, 2009.

43. Sackett $D$ L, Rosenberg W M C, Gray J A M, Haynes R B, Richardson W S. Evidence based medicine: what it is and what it isn't. Br Med J 1996 312: $71-72$.

44. Office for National Statistics. Children's Dental Health Survey [Online]. 2003. Available from: www. statistics.gov.uk/cci/nugget.asp?id=1000. 